\title{
CaMKII-Dependent Phosphorylation of the GTPase Rem2 Is Required to Restrict Dendritic Complexity
}

\author{
Amy E. Ghiretti, ${ }^{1,3}$ Katelyn Kenny, ${ }^{1,3}$ Michael T. Marr 2nd, ${ }^{2,3}$ and Suzanne Paradis ${ }^{1,3}$ \\ ${ }^{1}$ National Center for Behavioral Genomics and Volen Center for Complex Systems, ${ }^{2}$ Rosenstiel Basic Medical Sciences Research Center, and ${ }^{3}$ Department of \\ Biology, Brandeis University, Waltham, Massachusetts 02454
}

The morphogenesis of the dendritic arbor is a critical aspect of neuronal development, ensuring that proper neural networks are formed. However, the molecular mechanisms that underlie this dendritic remodeling remain obscure. We previously established the activityregulated GTPase Rem2 as a negative regulator of dendritic complexity. In this study, we identify a signaling pathway whereby Rem2 regulates dendritic arborization through interactions with $\mathrm{Ca}^{2+} /$ calmodulin-dependent kinases (CaMKs) in rat hippocampal neurons. Specifically, we demonstrate that Rem2 functions downstream of CaMKII but upstream of CaMKIV in a pathway that restricts dendritic complexity. Furthermore, we show that Rem2 is a novel substrate of CaMKII and that phosphorylation of Rem2 by CaMKII regulates Rem 2 function and subcellular localization. Overall, our results describe a unique signal transduction network through which Rem 2 and CaMKs function to restrict dendritic complexity.

\section{Introduction}

The development of the CNS encompasses a variety of complex processes, including the formation of synapses and the morphogenesis of the dendritic arbor. At the level of individual neurons, changes in these developmental processes are mediated in part by differences in gene expression and function (Horton and Ehlers, 2003; Jan and Jan, 2003; Loebrich and Nedivi, 2009; Lyons and West, 2011). Previously, we identified the activity-regulated GTPase Rem2 as a novel mediator of dendritic morphology in hippocampal neurons (Finlin et al., 2000; Paradis et al., 2007; Ghiretti and Paradis, 2011). Specifically, we showed that RNAimediated knockdown of Rem2 expression causes an increase in dendritic branching, demonstrating that the endogenous function of Rem2 is to inhibit dendritic complexity (Ghiretti and Paradis, 2011).

Rem2 is the only member of the Rad/Rem/Rem $2 / \mathrm{Gem} / \mathrm{Kir}$ (RGK) family of small Ras-like GTPases that is highly expressed in neurons (Finlin et al., 2000). Interestingly, the crystal structures of several RGK proteins, including Rem2, reveal key differences between this family and other Ras-like GTPases in their nucleotide binding domains, suggesting that Rem2 may not

\footnotetext{
Received Aug. 13, 2012; revised Feb. 27, 2013; accepted March 2, 2013.

Author contributions: A.E.G., M.T.M., and S.P. designed research; A.E.G. and K.K. performed research; A.E.G., K.K., M.T.M., and S.P. analyzed data; A.E.G. and S.P. wrote the paper.

This work was supported by the Alfred P. Sloan Foundation (S.P.), The Richard and Susan Smith Family Foundation (S.P), NIH Grant R01NS065856 (S.P.), NIH Grant F31DA032181 (A.E.G.), and NIH Grant P30NS45713 (for Core Facilities for Neurobiology at Brandeis University). We thank Dr. Thomas Soderling at Oregon Health \& Science University, Dr. Gary Wayman at Washington State University, and Dr. John Lisman at Brandeis University for generous sharing of reagents. We also thank Drs. Lisman and Gina Turrigiano for critical reading of this manuscript.

The authors declare no competing financial interests.

Correspondence should be addressed to Suzanne Paradis, Department of Biology, National Center for Behavioral Genomics, and Volen Center for Complex Systems, Brandeis University, 415 South Street, Waltham, MA 02454. E-mail: paradis@brandeis.edu.

DOI:10.1523/JNEUROSCI.3861-12.2013

Copyright $\odot 2013$ the authors $\quad 0270-6474 / 13 / 336504-12 \$ 15.00 / 0$
}

function as a classical GTPase regulated by its nucleotide binding state (Sasson et al., 2011; Reymond et al., 2012). Moreover, neither GTPase activating proteins nor guanine nucleotide exchange factors that regulate the activity of Rem 2 or other RGK proteins have been identified to date (Correll et al., 2008). Thus, regulation of Rem 2 function by posttranslational modification such as phosphorylation is an intriguing possibility. The effect of phosphorylation on Rem 2 has been studied previously in nonneuronal cell types: simultaneous phosphorylation of both S69 and S334 is required for an interaction between Rem 2 and 14-3-3 protein dimers (Béguin et al., 2005), which in turn affects the localization of Rem2 (Béguin et al., 2005; Ghiretti and Paradis, 2011). However, this finding has not been extended to neurons nor demonstrated to play a role in the function of Rem2.

Our previous work demonstrated that an interaction between Rem 2 and the calcium sensor calmodulin $(\mathrm{CaM})$ is required for Rem2 to restrict dendritic complexity (Ghiretti and Paradis, 2011). In addition, a number of studies suggest that calciumdependent signaling through $\mathrm{CaM}$ and several $\mathrm{Ca}^{2+} / \mathrm{CaM}$ dependent kinase (CaMK) family members, including CaMKI, CaMKII, and CaMKIV, both positively and negatively regulates dendritic complexity (Wu and Cline, 1998; Redmond et al., 2002; Vaillant et al., 2002; Fink et al., 2003; Wayman et al., 2004, 2006; Puram et al., 2011). Although the identity and function of downstream effectors of CaMK signaling that restrict dendritic morphology have not been elucidated, at least one RGK family member (Rad) has been demonstrated to be a CaMKII substrate in vitro (Moyers et al., 1998).

Therefore, we sought to determine whether Rem2 interacts with CaMKII and/or other CaMKs in a signal transduction cascade that regulates dendritic morphology. In this study, we report the first analysis of a Rem 2 signaling pathway in neurons and demonstrate that Rem 2 functions downstream of and is a novel substrate for CaMKII. Furthermore, we show that phosphoryla- 
tion of Rem 2 by CaMKII is required for Rem 2 to suppress dendritic complexity and to translocate to the nucleus, where it may function to suppress CaMKIV signaling. Overall, our results establish that at least one biological consequence of a signaling pathway involving CaMKs and Rem2 is to restrict dendritic arborization.

\section{Materials and Methods}

Plasmids and pharmacological reagents. GFP, Rem 2 shRNAs, and the RNAi-resistant Rem 2 cDNA were all described previously (Paradis et al., 2007; Ghiretti and Paradis, 2011). pEGFP-CaMKIV-nuc [as described by Wayman et al. (2006) and Schmitt et al. (2004)], pEGFP-CaMKI $\alpha$ (Wayman et al., 2004), and CaMKIIN (Wayman et al., 2004) constructs were a generous gift from Thomas Soderling (Oregon Health \& Sciences University). Constitutively active (CA) T286D/T305A/T306A CaMKII $\alpha$ and tCaMKII $\alpha$ (Pi et al., 2010) constructs were a generous gift from John Lisman (Brandeis University), and the QuikChange Site Directed $\mathrm{Mu}-$ tagenesis kit (Stratagene) was used on the T286D/T305A/T306A cDNA to generate a wild-type cDNA and then introduce the dominant-negative (DN) K42R mutation, which renders the protein kinase dead. CaMKII shRNA sequences were as described previously (Wheeler et al., 2008) and subcloned into the pSuper vector. W-7 and KN-93 were purchased from Sigma-Aldrich.

For the S-to-A Rem 2 cDNA constructs, we scanned the rat Rem2 protein sequence (NCBI reference sequence NP_073176.2) for the canonical CaMKII consensus phosphorylation site R-X-X-S/T (Kennelly and Krebs, 1991; Pearson and Kemp, 1991; Moyers et al., 1997) and identified six putative serine phosphorylation sites. The following Rem2 constructs were generated using the QuikChange Site Directed Mutagenesis kit (Stratagene) on the RNAi-resistant Rem 2 cDNA to change those serines to alanines. The forward primers used are listed, with the introduced mutations indicated in lowercase: S69A, CAGACGAAGAGGAgcC ATGCCCGTGCCCTAC; S241A, CCCGCTCCCGGGAGGTAgCACTG GAGGAGGGTC; S308A, CCTACGCGCCGTGAGgcCCTCACCAAGA AAGC; S334A, CAAGCAACGCTCCAGGgCATGTCACGACCTC.

Neuronal culture, transfection, and pharmacological treatment for morphology analysis. Neurons were cultured at low density on an astrocyte feeder layer, as described previously (Ghiretti and Paradis, 2011). Briefly, dissociated hippocampal neurons from E18 rats of either sex were plated in 24 -well plates at a density of $\sim 80,000$ per well onto a layer of confluent astrocytes and grown in Neurobasal medium with B27 supplement (Invitrogen).

Neurons were transfected by the calcium phosphate method (Xia et al., $1996)$ at 2 DIV. The relatively low transfection efficiency $(<10 \%$; our unpublished observations) of this method allows for a transfected neuron to develop in the context of a network of otherwise unaffected cells. Note that the cotransfection efficiency of up to three plasmids is $\sim 93 \%$ at both 14 DIV (Ghiretti and Paradis, 2011) and 5 DIV (data not shown).

All conditions were transfected with a GFP plasmid at $500 \mathrm{ng} /$ well. For control conditions, neurons were also transfected with empty pSuper vector at 33 or $100 \mathrm{ng} /$ well. For Rem 2 RNAi and rescue experiments, neurons were also transfected with a pSuper-shRNA plasmid containing an shRNA against Rem2 (Paradis et al., 2007) at 33 ng/well. For Rem2 overexpression and rescue experiments, neurons were also transfected with the RNAi-resistant Rem 2 cDNA or, in the case of the serine-toalanine mutation experiments, the relevant S-to-A Rem 2 mutant cDNA, at $100 \mathrm{ng} / \mathrm{well}$.

For experiments involving CaMKIV or CaMKI $\alpha$, each DN or CA CaMK mutant was transfected at $100 \mathrm{ng} /$ well, either alone or along with a Rem 2 shRNA at $33 \mathrm{ng} /$ well. DN and CA CaMKII $\alpha$ were transfected at $500 \mathrm{ng} /$ well, either alone or along with a Rem 2 shRNA at $33 \mathrm{ng} /$ well. The CaMKII shRNA was transfected at $33 \mathrm{ng} /$ well. CaMKIIN was overexpressed at $100 \mathrm{ng} /$ well.

For pharmacological experiments, neurons were also treated for $24 \mathrm{~h}$ before fixing with W-7 $(20 \mu \mathrm{M})$ or KN-93 $(20 \mu \mathrm{M})$.

Sholl analysis. At 4-5 DIV, the neuronal medium was replaced with $1 \times$ PBS, and neurons were fixed with $4 \%$ paraformaldehyde $/ 4 \%$ sucrose for $8 \mathrm{~min}$ at room temperature. Coverslips were then washed three times with $1 \times$ PBS for $5 \mathrm{~min}$ and mounted on glass slides for imaging. Twelvebit images were acquired on an Olympus Fluoview300 confocal microscope using a $20 \times$ oil objective. All images were acquired with identical settings for laser power, detector gain, and amplifier offset as a $z$-stack of 5-10 optical sections with a step size of $1.0 \mu \mathrm{m}$. For each experiment, images of 10-20 GFP-expressing neurons from at least two separate coverslips were acquired and analyzed for each condition, and each condition was analyzed in three independent experiments. All imaging and analysis was done in a blinded manner.

The Sholl analysis was performed on maximum intensity projections of each image by generating a series of 11 concentric circles of increasing radii (10 $\mu \mathrm{m}$ intervals) centered at the cell body, using the Image (NIH) plugin Concentric Circles. The number of dendrite crossings at each circle for each image was manually counted (Sholl, 1953), and the number of dendrite crossings at each circle for each transfection condition was calculated by averaging the number of crossings from every image. An experiment was deemed valid if the two control conditions (empty pSuper vector at 33 and $100 \mathrm{ng} /$ well) did not differ significantly. Subsequently, only one control was used for comparison with other transfection conditions.

Calf intestinal alkaline phosphatase treatment and Western blot. Cortices from E18 rats were homogenized on ice in RIPA buffer plus $1 \times$ Complete Protease Inhibitors (Roche) using a Dounce homogenizer. A Bio-Rad protein assay was used to determine total protein concentration, and equal amounts were used for all conditions in the assay. Three aliquots of homogenized cortex were used: one was immediately mixed with an equal volume of homemade $3 \times$ sample buffer (SDS, bromophenol blue, Tris, pH 6.8, glycerol, and $10 \% \beta$-mercaptoethanol) (control), a second was placed at $37^{\circ} \mathrm{C}$ for 30 min before mixing with an equal volume of sample buffer (mock), and the third was treated with $2 \mu \mathrm{l}$ of calf intestinal alkaline phosphatase (CIAP; Thermo Fisher Scientific) for $30 \mathrm{~min}$ at $37^{\circ} \mathrm{C}$ before mixing with an equal volume of sample buffer.

The lysates were boiled for $5 \mathrm{~min}$ and run on a 10\% SDS-PAGE gel to resolve proteins, and proteins were transferred to a nitrocellulose membrane. The membrane was probed with an in-house polyclonal antibody raised against rat Rem2 (1:100) (Ghiretti and Paradis, 2011) and anti- $\beta$-actin (1:5000; Abcam) as a loading control. Western blots were developed using the Odyssey Infrared Imaging System (Licor). The experiment was performed three times.

Recombinant protein purification. Plasmids containing GST or GSTtagged constructs (GST-Rem2, GST-Rem2 S241A/S308A, or GST-Rem2 S69A/S241A/S308A/S334A) were transformed into chemically competent BL21 Escherichia coli cells (Stratagene) and inoculated into $5 \mathrm{ml}$ of 2YT (tryptone, yeast extract, and $\mathrm{NaCl}, \mathrm{pH}$ 7.0) cultures. Cultures were grown shaking overnight at $37^{\circ} \mathrm{C}$ and diluted $1: 40$ in $2 \mathrm{YT}$ until they reached an $\mathrm{OD}_{600}$ between 0.4 and 0.6. The bacterial cultures were then induced with $0.5 \mathrm{mM} \mathrm{IPTG} \mathrm{(isopropyl} \beta$-D-1-thiogalactopyranoside) and grown shaking at $25^{\circ} \mathrm{C}$ overnight. The bacteria were pelleted and resuspended in resuspension buffer (20 mm Tris, $\mathrm{pH} 7.5,150 \mathrm{~mm} \mathrm{NaCl}, 1 \mathrm{~mm}$ DTT, $10 \%$ glycerol, and $1 \times$ Complete protease inhibitors; Roche). Lysozyme was added to a final concentration of $0.5 \mathrm{mg} / \mathrm{ml}$, and the reactions were incubated on ice for $20 \mathrm{~min}$. Deoxycholate and one-tenth the total volume of $5 \mathrm{M} \mathrm{NaCl}$ were added before sonication.

After sonication, the supernatant was isolated after centrifugation for $30 \mathrm{~min}$ at $15,000 \mathrm{rpm}$ and $4^{\circ} \mathrm{C}$. The supernatant was then added to Glutathione-Sepharose beads (GE Healthcare) and inverted for $1 \mathrm{~h}$ at $4^{\circ} \mathrm{C}$. The beads were pelleted and washed with wash buffer (50 mM Tris, $\mathrm{pH} 7.5,0.5 \mathrm{M} \mathrm{NaCl}, 1 \mathrm{~mm}$ DTT, $10 \%$ glycerol, and $1 \times$ Complete Protease Inhibitors; Roche). Recombinant proteins were eluted from beads by incubating for $30 \mathrm{~min}$ at $4^{\circ} \mathrm{C}$ with elution buffer [ $100 \mathrm{~mm}$ reduced glutathione (Sigma-Aldrich), $50 \mathrm{~mm}$ Tris, $\mathrm{pH}$ 6.8, with the $\mathrm{pH}$ adjusted to 8 using $1 \mathrm{~m} \mathrm{NaOH}$ ]. Total protein concentration was determined using a Bio-Rad protein assay.

In vitro kinase assay and Western blot. CaMKII $\alpha$ (New England Biologicals) was activated by incubation for $10 \mathrm{~min}$ at $30^{\circ} \mathrm{C}$ in an Eppendorf tube with the following: $12 \mu \mathrm{M}$ calmodulin, $20 \mathrm{~mm} \mathrm{CaCl}_{2}, 10 \mathrm{~mm}$ ATP, and $1 \times$ NEBuffer for protein kinases $\left(50 \mathrm{~mm}\right.$ Tris- $\mathrm{HCl}, 10 \mathrm{mM} \mathrm{MgCl}_{2}, 0.1$ mM EDTA, 2 mM DTT, and 0.01\% Brij 35). Five hundred units of activated CaMKII $\alpha$ were added to reaction tubes containing the following: 
distilled $\mathrm{H}_{2} \mathrm{O}\left(\mathrm{dH}_{2} \mathrm{O}\right), 10 \mu \mathrm{Ci}$ of EasyTides $\gamma^{-}{ }^{32} \mathrm{P}-\mathrm{ATP}$ (3000 Ci/mmol; PerkinElmer Life and Analytical Sciences), 2 mм ATP, $1 \times$ NEBuffer for protein kinases, and $1.5 \mu \mathrm{g}$ of a recombinant protein (GST, GST-Rem2, GST-Rem2 S241A/S308A, or GST-Rem2 S69A/S241A/S308A/S334A). The $20 \mu \mathrm{l}$ reactions were mixed and incubated at $30^{\circ} \mathrm{C}$ for $30 \mathrm{~min}$ and then on ice for $5 \mathrm{~min}$. Five microliters of homemade $3 \times$ sample buffer (6\% SDS, 0.1\% bromophenol blue, 150 mм Tris, pH 6.8, 30\% glycerol, and $10 \% \beta$-mercaptoethanol) were added to each reaction, and they were boiled for $5 \mathrm{~min}$. The completed reactions and a protein standard (Invitrogen) were electrophoresed on a 10\% SDS-PAGE gel. The gel was then stained with Coomassie Blue (50\% methanol, 10\% acetic acid, and 0.05\% Coomassie Brilliant Blue (Bio-Rad) for $1 \mathrm{~h}$, destained (10\% acetic acid, $50 \%$ methanol, $40 \% \mathrm{dH}_{2} \mathrm{O}$ ) overnight, and dried using a vacuum dryer for $1 \mathrm{~h}$ with heat. The dried gel was placed in a PhosphorImager cassette, exposed for $1 \mathrm{~h}$, and imaged using the STORM Molecular Imaging system.

For Western blotting to verify the identity of proteins, purified GST ( 1 $\mu \mathrm{g})$ and GST-Rem2 (0.5 or $1 \mu \mathrm{g})$ were combined with an equal volume of $3 \times$ sample buffer, boiled for $5 \mathrm{~min}$, and run on a 10\% SDS-PAGE gel. The Western blotting procedure was as described above; the nitrocellulose membrane was then probed with anti-Rem2 (1:100) and anti-GST (1:1000; Sigma-Aldrich).

Neuron culture, transfection, immunostaining, and analysis for shRNA verification and nuclear localization experiments. E18 rat hippocampal neurons were cultured and transfected at 2 DIV as described above with a GFP-expressing plasmid (500 ng/well) and the following: for shRNA verification, empty pSuper vector (100 ng/well), the shRNA against Rem2 used in morphology experiments (33 ng/well), or a second Rem2targeted shRNA (15 ng/well); for nuclear localization, empty pSuper vector (100 ng/well), CA T286D/T305A/T306A CaMKII $\alpha$ (500 ng/well), an S241A/S308A Rem2 cDNA (100 ng/well), or both CA CaMKII $\alpha$ and S241A/S308A Rem2. At 5 DIV, neuronal medium was replaced with $1 \times$ PBS, and neurons were fixed with $4 \%$ paraformaldehyde $/ 4 \%$ sucrose for $8 \mathrm{~min}$ at room temperature. Coverslips were then washed with $1 \times \mathrm{PBS}$ and incubated overnight with primary antibodies in a humidified chamber. For experiments to assess the efficacy of the Rem2-targeted shRNA, neurons were costained with rabbit anti-Rem2 (1:100; Ghiretti and Paradis, 2011) and mouse anti-MAP2 (1:1000; Sigma-Aldrich). For nuclear localization experiments visualizing endogenous Rem2, the primary antibody was rabbit anti-Rem2 (1:100; Ghiretti and Paradis, 2011). For experiments visualizing Rem2 S241A/S308A, the primary antibody was mouse anti-myc (1:500; Santa Cruz Biotechnology). All antibodies were prepared in $1 \times$ GDB $(0.1 \%$ gelatin, $0.3 \%$ Triton X-100, $4.2 \%$ of $0.4 \mathrm{M}$ phosphate buffer, and $9 \% 5 \mathrm{M} \mathrm{NaCl})$.

After overnight incubation, coverslips were washed three times with $1 \times$ PBS. Appropriate Cy3- or Cy5-conjugated secondary antibodies were diluted in $1 \times$ GDB and incubated on coverslips for $2 \mathrm{~h}$ at room temperature. Coverslips were washed once with $1 \times$ PBS for $10 \mathrm{~min}$. For nuclear localization experiments, DAPI ( $1: 1000)$ diluted in $1 \times$ PBS was added to coverslips and incubated at room temperature for $10 \mathrm{~min}$, followed by a final $1 \times$ PBS wash for $10 \mathrm{~min}$. Coverslips were dipped in $\mathrm{dH}_{2} \mathrm{O}$ and mounted on glass slides using Aquamount (Lerner Laboratories).

To assess the efficacy of our Rem2-targeted shRNA, 12-bit images of neurons were acquired on an Olympus Fluoview300 confocal microscope using a $60 \times$ oil objective. Within each experiment, identical settings for laser power, detector gain, and amplifier offset were used to acquire images. The $z$-stacks of $5-10$ optical sections with a step size of 0.5 $\mu \mathrm{m}$ were taken for 10-20 GFP-expressing neurons from three coverslips per condition for each experiment. Maximum intensity projections were created from the $z$-stacks. Each experiment was performed three times and imaged/analyzed by an experimenter blind to condition.

Staining intensity was assessed using MetaMorph image analysis software. First, a $25 \mu \mathrm{m}$ stretch of dendrite was identified and traced in the GFP channel. This stretch was then copied to both the Rem2 (to quantify Rem2 staining) and MAP2 (to quantify MAP2 staining), and average intensity was recorded. The staining intensity of the mock-transfected condition was compared with both RNAi conditions using SPSS software to run a two-way ANOVA (factors were transfection condition and experiment date) with Scheffé's post hoc test.
For nuclear localization experiments, 12-bit images of neurons were acquired on either an Olympus Fluoview300 confocal microscope using a $60 \times$ oil objective or on a Leica TCS SP 2 spectral confocal microscope using a $63 \times$ oil objective. Within each experiment, identical settings for laser power, detector gain, and amplifier offset were used to acquire images. The $z$-stacks of $5-10$ optical sections with a step size of $0.5 \mu \mathrm{m}$ were taken for 10-20 GFP-expressing neurons from three coverslips per condition for each experiment. Maximum intensity projections were created from the $z$-stacks. Each experiment was performed three times and imaged/analyzed by an experimenter blind to condition.

Nuclear and cytoplasmic staining intensity was quantified using MetaMorph image analysis software. First, the nuclear intensity of Rem2 (or myc) staining was determined in one of two ways: (1) the region of DAPI staining from a GFP-transfected neuron was traced and copied into the Rem2 (or myc) channel where the average pixel intensity of the nuclear staining was calculated, or (2) the Rem2 (or myc) channel was used to identify the nucleus within the soma of a GFP-transfected neuron by observation of the perturbation in staining caused by the nuclear membrane. We obtained the same results whether the Rem $2 /$ myc or DAPI staining was used to identify the nucleus. Next, the soma of the GFPtransfected neuron was traced and copied into the Rem 2 or myc channel, and the average pixel intensity of the total somatic staining was calculated. The value of the nuclear staining intensity was subtracted from the somatic staining intensity to yield a value for the cytoplasmic staining intensity. Nuclear staining intensity was then divided by the cytoplasmic staining intensity to yield a nuclear/cytoplasmic staining intensity ratio. The ratio for each condition was calculated by averaging the ratio for each image across all three experiments.

Statistics. For Sholl analysis, significance was determined using SPSS software to run a two-way multivariate ANOVA (factors were transfection condition and experiment date; circle radii were independent variables), followed by the Scheffé's post hoc test for significance at each circle radius. For shRNA verification, significance was determined by a twoway ANOVA (factors were transfection condition and experiment date). For nuclear localization, significance was determined by a pairwise ANOVA. For clarity and illustrative purposes, some data sets are plotted in more than one graph within a single figure, with the duplicate conditions indicated in the figure legends. These are Figure 1, $C$ and $D$ (mock and RNAi conditions); Figure 2, $A$ and $B$ (mock and RNAi conditions); Figure $3, A$ and $B$ (mock and RNAi conditions); Figure $4, A, B$, and $D$ (mock and RNAi conditions); and Figure 6B-D (mock and RNAi conditions). In every instance, at least three experiments were performed in parallel, and each experiment contained every condition. In addition, the data represented in these figures were compared as a single data set for statistical analysis, not individually for the conditions represented on each graph. Please refer to Tables 1-5 for complete statistical comparisons of all conditions within these single data sets.

\section{Results}

\section{Rem2 and CaMK signaling intersect to mediate dendritic complexity}

We initially investigated the interaction between Rem2 and CaMK signaling by pharmacologically manipulating CaMK activity alone or in combination with RNAi-mediated knockdown of Rem2 in cultured hippocampal neurons and by assaying the net effect on dendritic morphology. We transfected neurons at 2 DIV with a GFPexpressing plasmid and either an empty vector plasmid (Fig. 1 B1) or a plasmid expressing an shRNA against Rem2 (Fig. 1 B2). At 4 DIV, we treated neurons with $20 \mu \mathrm{M} \mathrm{W}-7$, a CaM blocker, or KN-93, a general CaMK blocker (Redmond et al., 2002; Wayman et al., 2004). At 5 DIV, we fixed and imaged these neurons and assessed dendritic complexity via Sholl analysis (see Fig. $1 B-D$ ), which involves superimposing a series of concentric circles of increasing diameter, centered at the soma, on a low-magnification image of a neuron and quantifying the number of dendrites that intersect these circles (Sholl, 1953). The efficacy of the Rem 2 shRNA after $48 \mathrm{~h}$ of transfection was verified in both HEK 293T cells (Ghiretti and Paradis, 2011) 
Table 1. Statistical significance for all conditions compared in the single data set represented in Figure 1

\begin{tabular}{lcccccc}
\hline & Mock & Mock + W-7 & Mock + KN-93 & RNAi & RNAi + W-7 & RNAi + KN-93 \\
\hline Number & 46 & 23 & 34 & 45 & 21 & 32 \\
Mock & & $* *$ & $*$ & $*$ & $* *$ & NS \\
Mock + W-7 & $* *$ & & NS & $* * *$ & NS & $* *$ \\
Mock + KN-93 & $*$ & NS & & $* *$ & NS & $*$ \\
RNAi & $*$ & $* * *$ & $* *$ & & $* * *$ & $*$ \\
RNAi + W-7 & $* *$ & NS & NS & $* * *$ & & $* *$ \\
RNAi + KN-93 & NS & $* *$ & $*$ & $*$ & $* *$ &
\end{tabular}

In Tables 1-5: Statistical tables showing the total number of neurons per condition and the significance of all conditions from one another for all experiments/figures in which multiple graphs are used to represent data. ${ }^{*} p<$ 0.05 ; ${ }^{* *} p<0.01 ;{ }^{* * *} p<0.001$. NS, Not significant. Data within each table are the combination of three to four independent experiments, each of which contained every condition within that table. See figure legends for the statistical test used.

Table 2. Statistical significance for all conditions compared in the single data set represented in Figure 2

\begin{tabular}{lcccccc}
\hline & Mock & RNAi & CA CaMKIV & DN CaMKIV & RNAi + CA & RNAi + DN \\
\hline Number & 44 & 48 & 52 & 43 & 51 & 42 \\
Mock & & $*$ & $*$ & NS & $*$ & NS \\
RNAi & $*$ & & NS & $*$ & NS & $*$ \\
CA CaMKIV & $*$ & NS & & $*$ & NS & $*$ \\
DN CaMKIV & NS & $*$ & $*$ & & $*$ & NS \\
RNAi + CA & $*$ & NS & NS & $*$ & & $*$ \\
RNAi + DN & NS & $*$ & $*$ & NS & $*$ & NS \\
\hline
\end{tabular}

Table 3. Statistical significance for all conditions compared in the single data set represented in Figure 3

\begin{tabular}{lcccccc}
\hline & Mock & RNAi & CA CaMKI & DN CaMKI & RNAi + CA & RNAi + DN \\
\hline Number & 55 & 48 & 56 & 57 & 46 & 35 \\
Mock & & $*$ & $*$ & NS & $* *$ & NS \\
RNAi & $*$ & & NS & $*$ & $*$ & $*$ \\
CA CaMKI & $*$ & NS & & $*$ & $* *$ & $*$ \\
DN CaMKI & NS & $*$ & $*$ & & $* *$ & NS \\
RNAi + CA & $* *$ & $*$ & $*$ & $* *$ & & $* *$ \\
RNAi + DN & NS & $*$ & $*$ & NS & $* *$ & \\
\hline
\end{tabular}

Table 4. Statistical significance for all conditions compared in the single data set represented in Figure $4, A, B$, and $D$

\begin{tabular}{lccccccc}
\hline & Mock & RNAi & CA CaMKII & DN CaMKII & RNAi + CA & RNAi + DN & CaMKIIN \\
\hline Number & 49 & 42 & 38 & 30 & 38 & 45 & 32 \\
Mock & & $*$ & $*$ & $*$ & $*$ & $*$ & $*$ \\
RNAi & $*$ & & $* *$ & NS & NS & NS & NS \\
CA CaMKII & $*$ & $* *$ & & $* *$ & $* *$ & $* *$ & $*$ \\
DN CaMKII & $*$ & NS & $* *$ & & NS & NS & NS \\
RNAi + CA & $*$ & NS & $* *$ & NS & & NS & NS \\
RNAi + DN & $*$ & NS & $* *$ & NS & NS & & NS \\
CaMKIIN & $*$ & NS & $*$ & NS & NS & NS & \\
\hline
\end{tabular}

and cortical neurons (data not shown) via Western blotting. Further, specificity was verified by both rescue experiments (Ghiretti and Paradis, 2011) and observation of identical phenotypes with a second Rem2-targeted shRNA that can also be rescued (data not shown). In addition, we observed a 50\% decrease in Rem2 staining intensity via immunocytochemistry in 5 DIV hippocampal neurons transfected with two independent shRNAs targeting Rem2, convincing evidence that Rem 2 expression is significantly decreased at this time point (Fig. 1A).

Interestingly, we found that treatment of mock transfected neurons with either $\mathrm{W}-7$ or $\mathrm{KN}-93$ led to decreased complexity compared with untreated mock transfected neurons (Fig. 1, compare $B 1$ with $B 3$ and $B 5$; quantified in Fig. $1, C$ and $D$ ), in agreement with previous work (Fink et al., 2003). W-7 or KN-93 treatment also suppressed the Rem2 RNAi-induced increase in complexity (Fig. 1, compare B2 with $B 4$ and B6; quantified in Fig. $1, C$ and $D$ ), suggesting a number of nonmutually exclusive possibilities about the intersection between Rem 2 and CaMK signaling. For example, it could be that Rem 2 functions downstream of, and is positively regulated by, one or more CaMKs. Another possibility is that the increase in complexity seen with Rem 2 RNAi is attributable to the increased activation of an opposing CaMK signaling pathway that promotes complexity. However, it should be noted that KN-93 is a broad-spectrum inhibitor with a number of molecular targets (Enslen et al., 1994; Ledoux et al., 1999; Gao et al., 2006), and thus these results do not identify the specific CaMKs that mediate these effects. Broadly speaking, these results do suggest that regulation of dendritic complexity in general occurs mainly through CaM-mediated signaling pathways.

\section{Identification of specific CaMKs that interact with Rem2}

Several CaMK family members have been previously implicated as either promoting or inhibiting dendritic complexity (Wu and Cline, 1998; Redmond et al., 2002; Vaillant et al., 2002; Fink et al., 2003; Wayman et al., 2004, 2006; Puram et al., 2011). We chose to focus specifically on CaMKIV, CaMKI $\alpha$, and CaMKII signaling pathways for several reasons. First, Rem2 is widely expressed in neurons (Ghiretti and Paradis, 2011), including in the nucleus (Béguin et al., 2005; Mahalakshmi et al., 2007; Ghiretti and Paradis, 2011), whereas CaMKIV is constitutively found in the nucleus, where it mediates CREB-dependent gene transcription (Matthews et al., 1994). Second, CaMKI $\alpha$ has been reported to lie downstream of neuronal depolarization in mediating dendritic complexity (Wayman et al., 2006, 2011), and our pharmacological studies also implicate Rem2 in this process (A. E. Ghiretti, A. R. Moore, and S. Paradis, unpublished observations). Finally, the RGK family member Rad is a substrate of CaMKII in vitro

Table 5. Statistical significance for all conditions compared in the single data set represented in Figure 6

\begin{tabular}{|c|c|c|c|c|c|c|c|c|c|c|c|}
\hline & Mock & RNAi & Rescue & S69A 0E & S69A Rescue & S241A OE & S241A Rescue & S308A 0E & S308A Rescue & S334A OE & S334A Rescue \\
\hline Number & 52 & 45 & 58 & 55 & 48 & 44 & 41 & 48 & 44 & 54 & 42 \\
\hline Mock & & * & NS & NS & NS & * & * & NS & * & NS & NS \\
\hline RNAi & * & & * & * & * & NS & NS & * & NS & * & * \\
\hline S69A OE & NS & * & NS & & NS & * & * & NS & * & NS & NS \\
\hline S69A Rescue & NS & * & NS & NS & & * & * & NS & * & NS & NS \\
\hline S241A OE & * & NS & * & * & * & & NS & * & NS & * & * \\
\hline S308A Rescue & * & NS & * & * & * & NS & NS & * & & * & * \\
\hline $2334 \mathrm{~A} 0 \mathrm{E}$ & NS & * & NS & NS & NS & * & * & NS & * & & NS \\
\hline S334A Rescue & NS & * & NS & NS & NS & * & * & NS & * & NS & \\
\hline
\end{tabular}


A
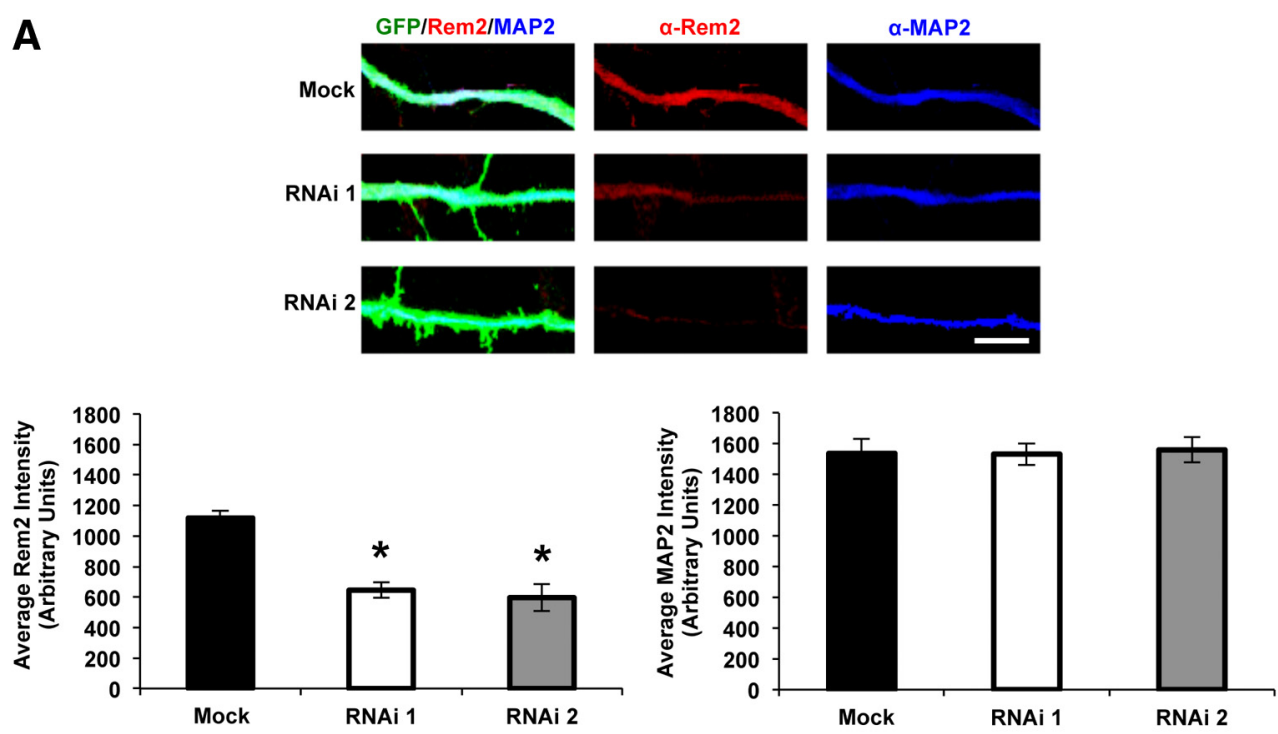

B
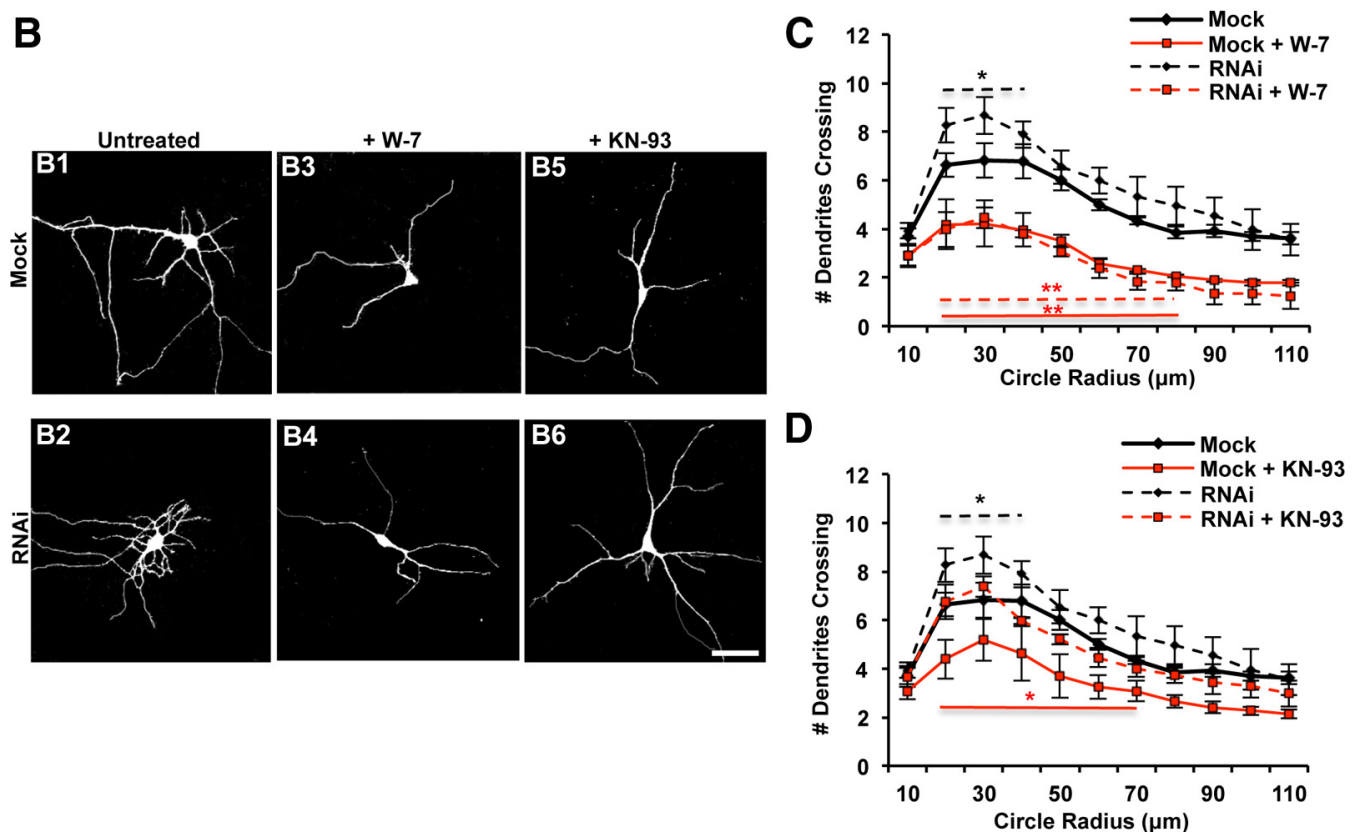

Figure 1. Rem2 and CaMK signaling pathways interact to regulate dendritic morphology. $A$, Representative images (top) and quantification of staining intensity (bottom) of hippocampal neurons transfected at 2 DIV with GFP and an empty vector (Mock), the Rem2 shRNA used in subsequent morphology experiments (RNAi 1), or a second Rem2 2 shRNA (RNAi 2). At 5 DIV, the neurons were fixed and stained for Rem 2 and MAP2. ${ }^{*} p<0.05$, two-way ANOVA with Scheffé's post hoc test. $n>50$ neurons per condition. Scale bar, $5 \mu \mathrm{m}$. $\boldsymbol{B}$, Representative images of 5 DIV neurons transfected with a GFP-expressing plasmid (Mock) or a GFP-expressing plasmid plus a Rem2 shRNA (RNAi) and then untreated or treated for $24 \mathrm{~h}$ with $20 \mu \mathrm{m} \mathrm{W}-7$ or $20 \mu \mathrm{m} \mathrm{KN-93}$. Unless otherwise indicated, in this and all subsequent figures, scale bars represent $50 \mu \mathrm{m}$. C, Dendritic complexity of 5 DIV mock and Rem2 RNAi neurons with or without $20 \mu \mathrm{M} \mathrm{W}-7$ treatment for $24 \mathrm{~h}$. In this and all subsequent figures, complexity is quantified via Sholl analysis, and a bar above or below the curve indicates significance at those particular radii compared with mock. Bars are colored and/or dashed to match the style/color of the data curve to which they refer. D, Dendritic complexity of 5 DIV mock and Rem2 RNAi neurons with or without $20 \mu \mathrm{m} \mathrm{KN}-93$ treatment for $24 \mathrm{~h}$. Here, mock and RNAi data from C are replotted for ease of comparison with other conditions. ${ }^{*} p<0.05$; ${ }^{* *} p<0.01$, multivariate ANOVA with Scheffé's post hoc test. $n=21-46$ neurons per condition.

(Moyers et al., 1997, 1998), and we hypothesized that Rem2 might be as well.

Therefore, we used well characterized CA, DN, or RNAi constructs to alter the activity of these CaMKs (see Materials and Methods for details) to determine whether Rem2 interacts with these specific CaMK family members. In general, the experiments were performed as follows. At 2 DIV, neurons were cotransfected with GFP and an shRNA construct against Rem2 alone ("RNAi"), a CA or DN CaMK construct alone ("CA CaMK" or "DN CaMK"), a shRNA construct against CaMKII alone ("CaMKII RNAi"), or a combination of the Rem2 shRNA and CaMK construct ("RNAi + CA CaMK" or "RNAi + DN CaMK" or "Rem2
RNAi + CaMKII RNAi"). At 5 DIV, we fixed and imaged these neurons and assessed their dendritic morphology via Sholl analysis (Sholl, 1953).

\section{CaMKIV}

The single CaMKIV gene has been implicated in promoting activity-dependent increases in dendritic complexity (Redmond et al., 2002). We overexpressed a CA CaMKIV, whose localization is restricted to the nucleus by a nuclear localization signal (Wayman et al., 2006, 2011), and observed a significant increase in dendritic complexity, suggesting that CaMKIV acts to promote this process (Fig. $2 \mathrm{~A}$ ), consistent with previous results (Redmond 
A

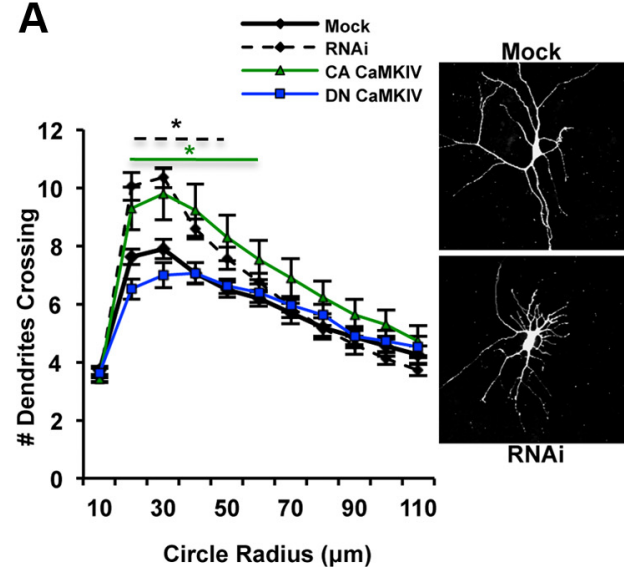

B

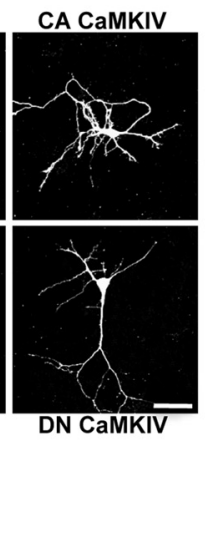

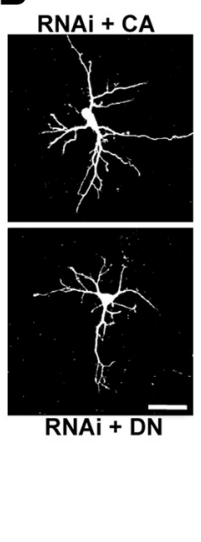

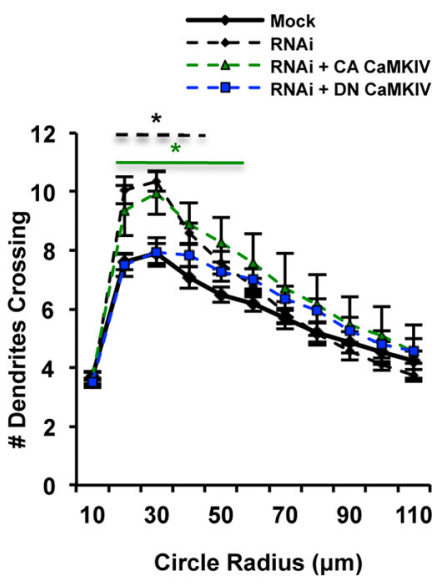

Figure 2. Rem2 is upstream of CaMKIV in a signaling pathway that regulates dendritic complexity. A, Quantification (left) and representative images (right) of dendritic complexity at 5 DIV of mock transfected neurons compared with Rem2 RNAi-, CA CaMKIV-, and DN CaMKIV-transfected neurons. $\boldsymbol{B}$, Representative images (left) and quantification (right) of dendritic complexity at 5 DIV of mock and Rem2 RNAi-transfected neurons compared with neurons cotransfected with both Rem2 RNAi and either CA or DN CaMKIV. Mock and RNAi data from $\boldsymbol{A}$ are replotted for ease of comparison with other conditions. ${ }^{*} p<0.05$, multivariate ANOVA with Scheffé's post hoc test. $n=42-52$ neurons per condition.

A

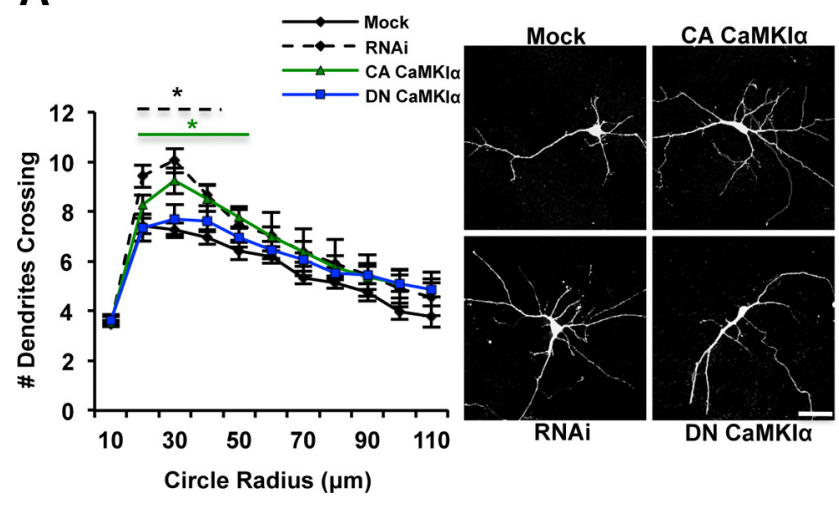

B

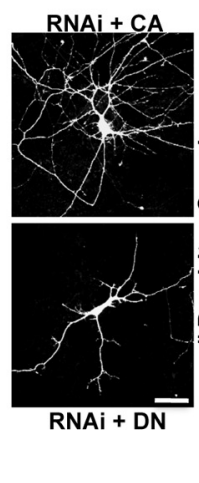

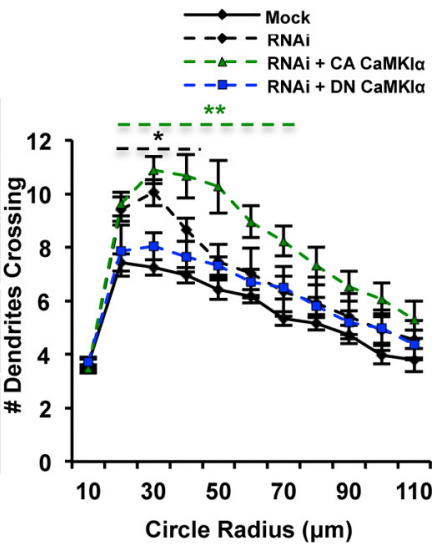

Figure 3. Rem2 signals in parallel to a CaMKl $\alpha$ pathway to mediate dendritic complexity. A, Quantification (left) and representative images (right) of dendritic complexity at 5 DIV of mock transfected neurons compared with Rem2 RNAi-, CA CaMKI $\alpha$-, and DN CaMKI $\alpha$-transfected neurons. B, Representative images (left) and quantification (right) of dendritic complexity at 5 DIV of mock and Rem2 RNAi-transfected neurons compared with neurons cotransfected with both Rem2 RNAi and either CA or DN CaMKI $\alpha$. Mock and RNAi data from $A$ are replotted for ease of comparison with other conditions. ${ }^{*} p<0.05,{ }^{* *} p<0.01$, multivariate ANOVA with Scheffé's post hoc test. $n=35-57$ neurons per condition.

et al., 2002). Overexpression of DN CaMKIV did not result in a significant change in dendritic complexity compared with mock transfected neurons (Fig. 2A), also consistent with previous results (Redmond et al., 2002; Wayman et al., 2006, 2011). The concomitant RNAi-mediated knockdown of Rem2 and overexpression of CA CaMKIV did not have an additive effect: whereas these neurons were highly complex, the effect was no larger than that of either Rem2 RNAi or CA CaMKIV alone (Fig. 2 B). However, overexpression of DN CaMKIV was able to suppress the Rem2 RNAi phenotype, causing these neurons to resemble those expressing DN CaMKIV alone (Fig. 2B). Together, these results suggest a signaling pathway in which CaMKIV lies downstream of, and is inhibited by, Rem 2 to mediate dendritic morphology.

\section{CaMKI $\alpha$}

The $\alpha$ isoform of CaMKI, which has a predominantly cytoplasmic localization, has been shown to promote both axonal and dendritic complexity in a neuronal activity-dependent manner (Takemoto-Kimura et al., 2003; Wayman et al., 2004, 2006; Ageta-Ishihara et al., 2009). Consistent with previous results, we observed that overexpression of CA CaMKI $\alpha$ alone significantly increased dendritic complexity (Fig. 3A), supporting the conclu- sion that CaMKI $\alpha$ acts to promote this process. Additionally, overexpression of DN CaMKI $\alpha$ alone did not result in a change in dendritic complexity (Fig. 3A), also consistent with previous results (Wayman et al., 2004, 2006, 2011). Interestingly, neurons expressing both Rem 2 RNAi and CA CaMKI $\alpha$ showed an additive increase in dendritic complexity, with a more complex dendritic phenotype than neurons expressing either Rem2 RNAi or CA CaMKI $\alpha$ alone (Fig. 3B). This additive effect suggests that CaMKI $\alpha$ and Rem 2 signal in parallel to have opposing effects on dendritic complexity. However, coexpression of DN CaMKI $\alpha$ with Rem2 RNAi produced a phenotype most similar to that of neurons transfected with DN CaMKI $\alpha$ alone (Fig. $3 B$ ), a result that is more consistent with CaMKI $\alpha$ functioning downstream of Rem2. Together, our results suggest that CaMKI $\alpha$ signals to increase dendritic complexity, whereas Rem 2 and CaMKIV signal in a parallel pathway that ultimately limits complexity.

\section{CaMKII}

A single active CaMKII holoenzyme consists of two hexamer rings composed of either CaMKII $\alpha$ isoform homomers or CaMKII $\alpha$ and CaMKII $\beta$ isoform heteromers (Lisman et al., 2002, 2012). CaMKII has been shown to regulate dendritic mor- 
A
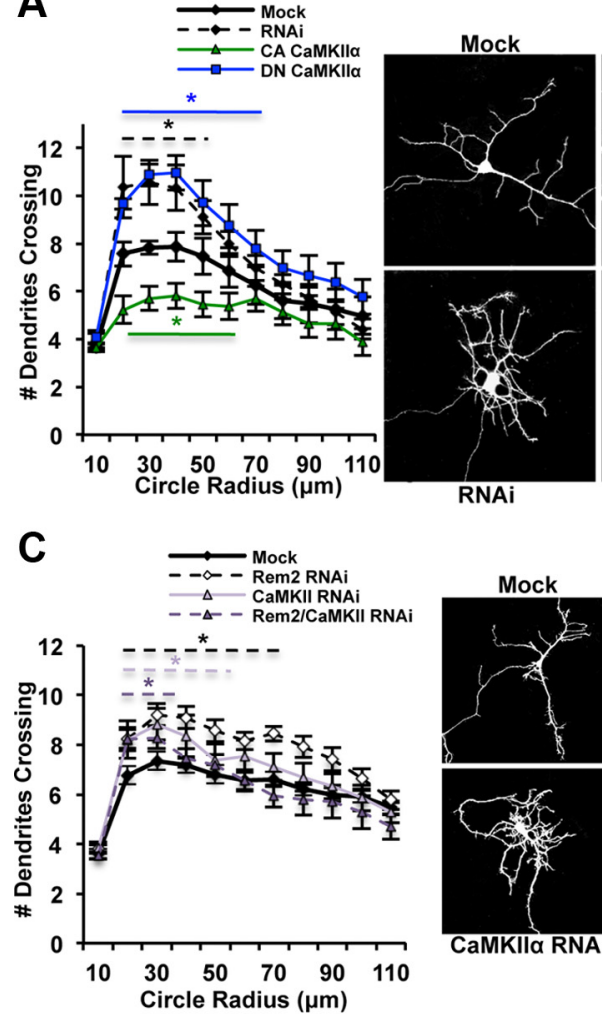
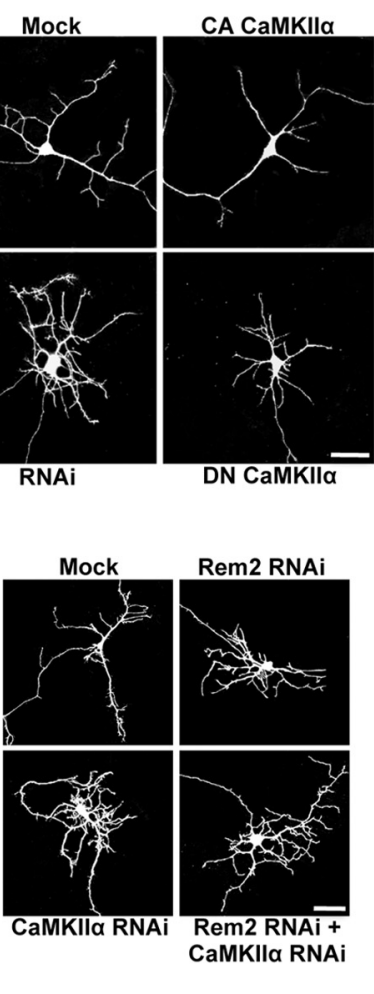

B
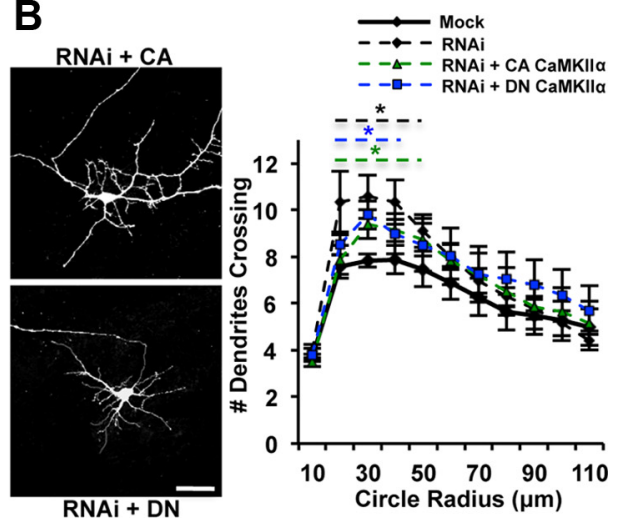

D
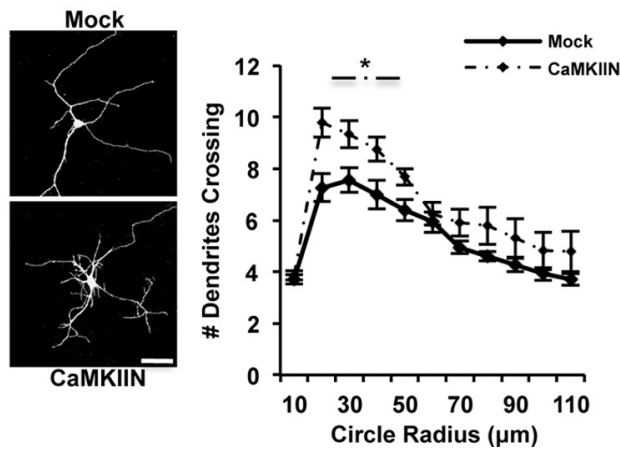

Figure 4. CaMKII signals through Rem2 to inhibit dendritic complexity. $A$, Quantification (left) and representative images (right) of dendritic complexity at 5 DIV of mock transfected neurons compared with Rem2 RNAi-, CA CaMKIl $\alpha$-, and DN CaMKIl $\alpha$-transfected neurons. B, Representative images (left) and quantification (right) of dendritic complexity at 5 DIV of mock and Rem2 RNAi-transfected neurons compared with neurons cotransfected with both Rem2 RNAi and either CA or DN CaMKII $\alpha$. Mock and RNAi data from $A$ are replotted for ease of comparison with other conditions. C, Quantification (left) and representative images (right) of dendritic complexity at 5 DIV of mock transfected neurons compared with Rem2 RNAi and CaMKIl $\alpha$ RNAi, alone or in combination as indicated, transfected neurons. D, Representative images (left) and quantification (right) of dendritic complexity at 5 DIV of mock transfected neurons compared with neurons expressing the CaMKII-specific peptide inhibitor CaMKIIN. Mock data from $A$ are replotted for ease of comparison with other conditions. ${ }^{*} p<0.05$, multivariate ANOVA with Scheffe's post hoc test. $n=30-49$ neurons per condition.

phology in a variety of experimental systems, although results have not been consistent between various studies (Wu and Cline, 1998; Fink et al., 2003; Gaudillière et al., 2004; Puram et al., 2011). These inconsistencies are possibly caused by the use of different approaches to manipulate CaMKII activity or different neuronal subtypes. For example, RNAi-mediated knockdown of CaMKII $\alpha$ in vitro in the rodent cerebellum leads to a decrease in complexity, implicating CaMKII as a promoter of dendritic complexity (Gaudillière et al., 2004). In contrast, overexpression of truncated CA CaMKII $\alpha$ mutants in Xenopus laevis tadpoles or in vitro in rodent hippocampal neurons leads to a decrease in complexity, suggesting that CaMKII is a negative regulator of dendritic arbor growth (Wu and Cline, 1998; Fink et al., 2003). Thus, we chose to investigate the interaction between Rem 2 and CaMKII activity using $\mathrm{CA}, \mathrm{DN}$, and RNAi constructs targeting the most highly expressed CaMKII isoform in hippocampus, CaMKII $\alpha$ (Lisman et al., 2002). These approaches should affect most, if not all, CaMKII-dependent activity in our hippocampal neurons.

We found that overexpression of autophosphorylated CA CaMKII $\alpha$ (T286D/T305A/T306A) (Pi et al., 2010) led to a decrease in dendritic complexity (Fig. 4A). We also observed this same phenotype after overexpression of a truncated, constitutively active form of the protein (tCaMKII $\alpha$ ) (data not shown), which lacks the autoinhibitory domain and has previously been used to demonstrate a decrease in complexity ( $\mathrm{Wu}$ and Cline, 1998). Consistent with these results, overexpression of kinase dead DN CaMKII $\alpha$ (K42R) led to a significant increase in complexity (Fig.
$4 A)$. Together, these results suggest that CaMKII activity inhibits dendritic complexity. Interestingly, when either CA or DN CaMKII $\alpha$ were overexpressed together with Rem2 RNAi, the resulting phenotype was increased complexity similar to that of Rem2 RNAi alone (Fig. 4B). Based on these observations, we conclude that Rem 2 lies downstream of CaMKII and that CaMKII signals through Rem2 to inhibit dendritic complexity.

As an additional control, we compared the phenotypes observed with DN CaMKII $\alpha$ with those observed with RNAimediated knockdown of CaMKII $\alpha$ expression, using a previously validated shRNA construct (Wheeler et al., 2008). We observed that RNAi-mediated knockdown of CaMKII $\alpha$ led to an increase in complexity both with and without RNAi of Rem2 (Fig. 4C), consistent with our observations using the DN construct (Fig. $4 A$ ). Similarly, the effect of DN CaMKII $\alpha$ was recapitulated in neurons expressing the CaMKII-specific inhibitory peptide CaMKIIN (Fig. 4D). CaMKIIN is a naturally occurring peptide that binds specifically to the catalytic domain of CaMKII (and not other CaMKs), potently inhibiting its kinase activity (Chang et al., 1998). Based on these observations, we conclude that Rem2 lies downstream of CaMKII and that CaMKII signals through Rem2 to inhibit dendritic complexity.

\section{Rem2 is a novel substrate for CaMKII}

The function and localization of RGK proteins is subject to regulation by phosphorylation (Correll et al., 2008), and a number of studies have observed that Rem 2 is detected as a doublet by 

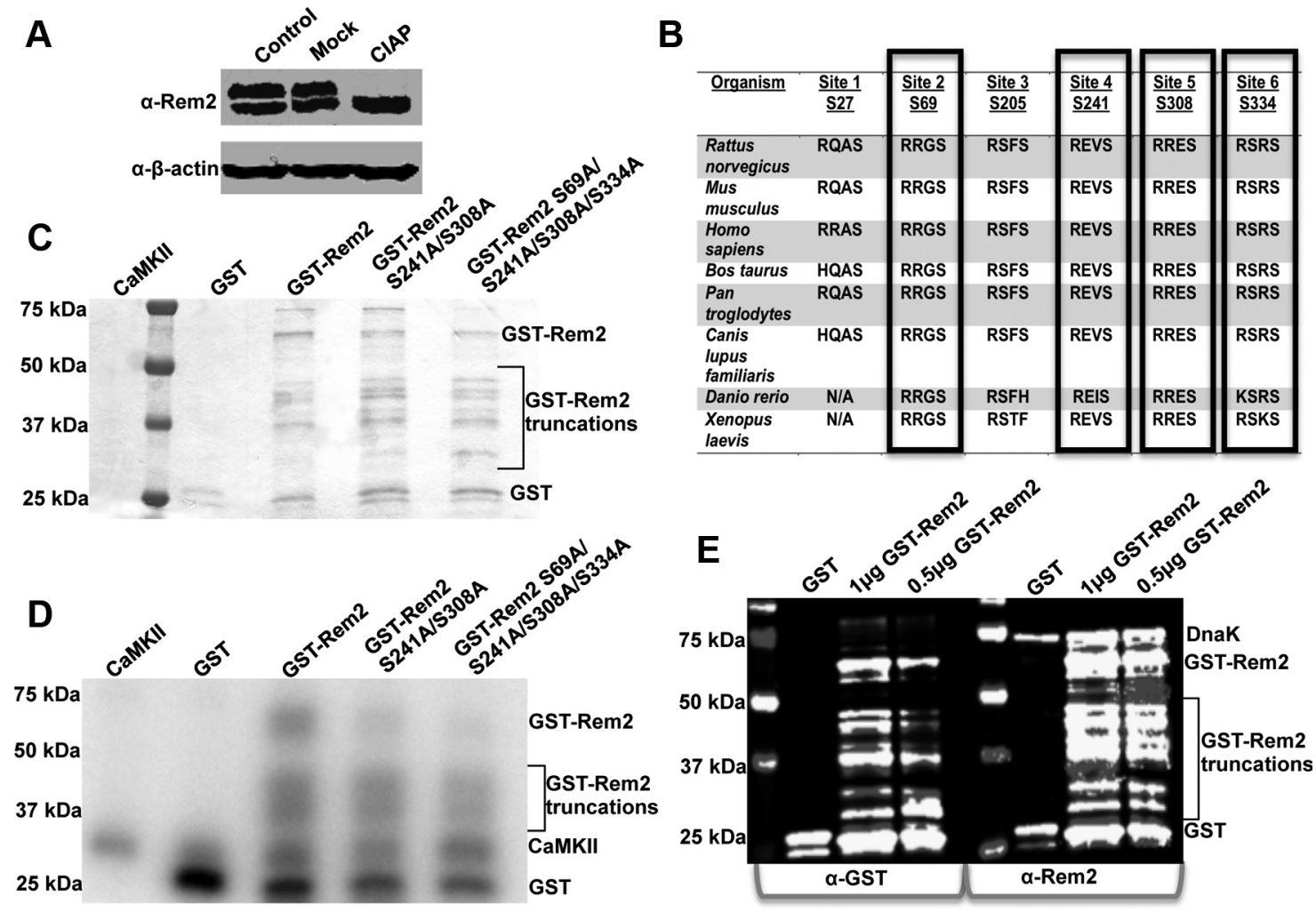

Figure 5. Rem2 is a phosphoprotein with six putative CaMKII phosphorylation sites. A, Western blot of lysates from E18 rat cortical neuron lysates that were (from left) untreated (Control), mock treated (Mock), or treated with CIAP for $30 \mathrm{~min}$. The blot was probed with a polyclonal antibody against rat Rem2 (Ghiretti and Paradis, 2011) and an antibody against $\beta$-actin as a loading control. B, Alignment of the CaMKII consensus phosphorylation sites in the rat Rem2 protein with other vertebrate Rem2 homologs, demonstrating that putative CaMKII phosphorylation sites 2, 4, 5, and 6 are highly conserved in vertebrates. C, Coomassie-stained SDS-PAGE gel depicting the peptides present in an in vitro kinase assay containing ATP $\gamma \mathrm{P}^{32}$ and (left to right) activated CaMKII alone or activated CaMKII plus purified GST, purified GST-Rem2, purified GST-Rem2 S241A/S308A, or purified GST-Rem2 S69A/S241A/S308A/S334A. Note that the intensity of the $65 \mathrm{kDa}$ band corresponding to GST-Rem2 constructs is comparable across all lanes, demonstrating equal protein loading. $\boldsymbol{D}$, Autoradiograph of the gel in Cidentifying proteins phosphorylated by CaMKII. GST-Rem2 runs at 65 $\mathrm{kDa}$, and decreasing phosphorylation is observed with S241A/S308A and S69A/S241A/S308A/S334A mutants. Note that autophosphorylated CaMKII is detected in all lanes (30 kDa) as is phosphorylated GST ( $26 \mathrm{kDa}$ ). E, Western blot demonstrating that the peptides between 26 and $65 \mathrm{kDa}$ detected by Coomassie stain in Cand by autoradiography in D represent GST-Rem 2 truncation products, as they are detected by both anti-GST and anti-Rem2 antibodies, along with full-length GST-Rem2 (65 kDa). Note that GST ( $26 \mathrm{kDa}$ ) is also detected by both anti-GST and anti-Rem2 antibodies as the Rem2 antibody was raised against GST-Rem2 in rabbits (Ghiretti and Paradis, 2011). The high-molecular-weight band (70 kDa) is DnaK, a bacterial contaminant from the GST or GST-Rem2 protein purification process.

Western blotting (Finlin et al., 2000; Béguin et al., 2005, 2007; Paradis et al., 2007; Ghiretti and Paradis, 2011). We observed that alkaline phosphatase treatment of E18 rat cortical lysates collapsed the Rem 2 doublet to a single band, leading us to conclude that Rem2 is a phosphoprotein in neurons (Fig. 5A). We identified six CaMKII consensus phosphorylation sites, R-X-X-S/T (Kennelly and Krebs, 1991; Pearson and Kemp, 1991; Moyers et al., 1997), in the rat Rem2 coding sequence, four of which are conserved in vertebrates (Fig. 5B): S69, S241, S308, and S334.

Given this high level of conservation, we asked whether any of these sites could be phosphorylated by CaMKII using an in vitro kinase assay. We purified GST-tagged wild-type Rem2 (GSTRem2) or GST-Rem2 in which a combination of putative phosphorylation sites had been mutated to alanines (e.g., GST-Rem2 S241A/S308A and GST-Rem2 S69A/S241A/S308A/S334A) from E. coli and incubated the purified Rem2 proteins with activated CaMKII and radiolabeled ATP. Separation by electrophoresis of equal amounts of each reaction followed by phosphorimaging revealed a radiolabeled band of $\sim 65 \mathrm{kDa}$ in the GST-Rem2 lane corresponding to full-length GST-Rem2 (Fig. 5D). Notably, the labeling was significantly reduced in the reactions containing the GST-Rem2 S241A/S308A protein and even further reduced in reactions containing the GST-Rem2 S69A/S241A/S308A/S334A protein. Our Coomassie-stained gel (Fig. $5 \mathrm{C}$ ) revealed a number of lower-molecular-weight bands that were identified as Rem2degradation products after immunoblotting with a rabbit antiRem2 antibody and a mouse anti-GST antibody (Fig. 5E). These results allow us to draw two important conclusions. First, our data indicate that Rem2 is a CaMKII substrate in vitro. Second, Rem2 is phosphorylated by CaMKII at S241 and S308 and, in addition, is phosphorylated at S69 and/or S334.

To test the functional relevance of phosphorylation of Rem2 at these serine residues, we introduced serine-to-alanine mutations to abolish potential phosphorylation at each of these sites into an RNAi-resistant Rem 2 cDNA. At 2 DIV, we cotransfected hippocampal neurons with plasmids expressing GFP, a Rem2 shRNA, and either a wild-type RNAi-resistant Rem 2 cDNA or an RNAi-resistant Rem 2 cDNA with the relevant S-to-A mutations. At 5 DIV, we fixed and imaged these neurons and assessed their dendritic complexity via Sholl analysis. We found that much like a wild-type RNAi-resistant Rem2 cDNA (which contains no serine-to-alanine mutations; Fig. 6A, B), S69A and S334A RNAiresistant Rem 2 were both able to rescue the increase in complexity as a result of Rem2 RNAi (Fig. 6A,C,D). In contrast, S241A and S308A mutations rendered the RNAi-resistant Rem 2 cDNA incapable of rescuing the RNAi phenotype (Fig. $6 A, C, D$ ). Thus, CaMKII phosphorylation at sites S241 and S308 is required for Rem2 to mediate dendritic complexity. 


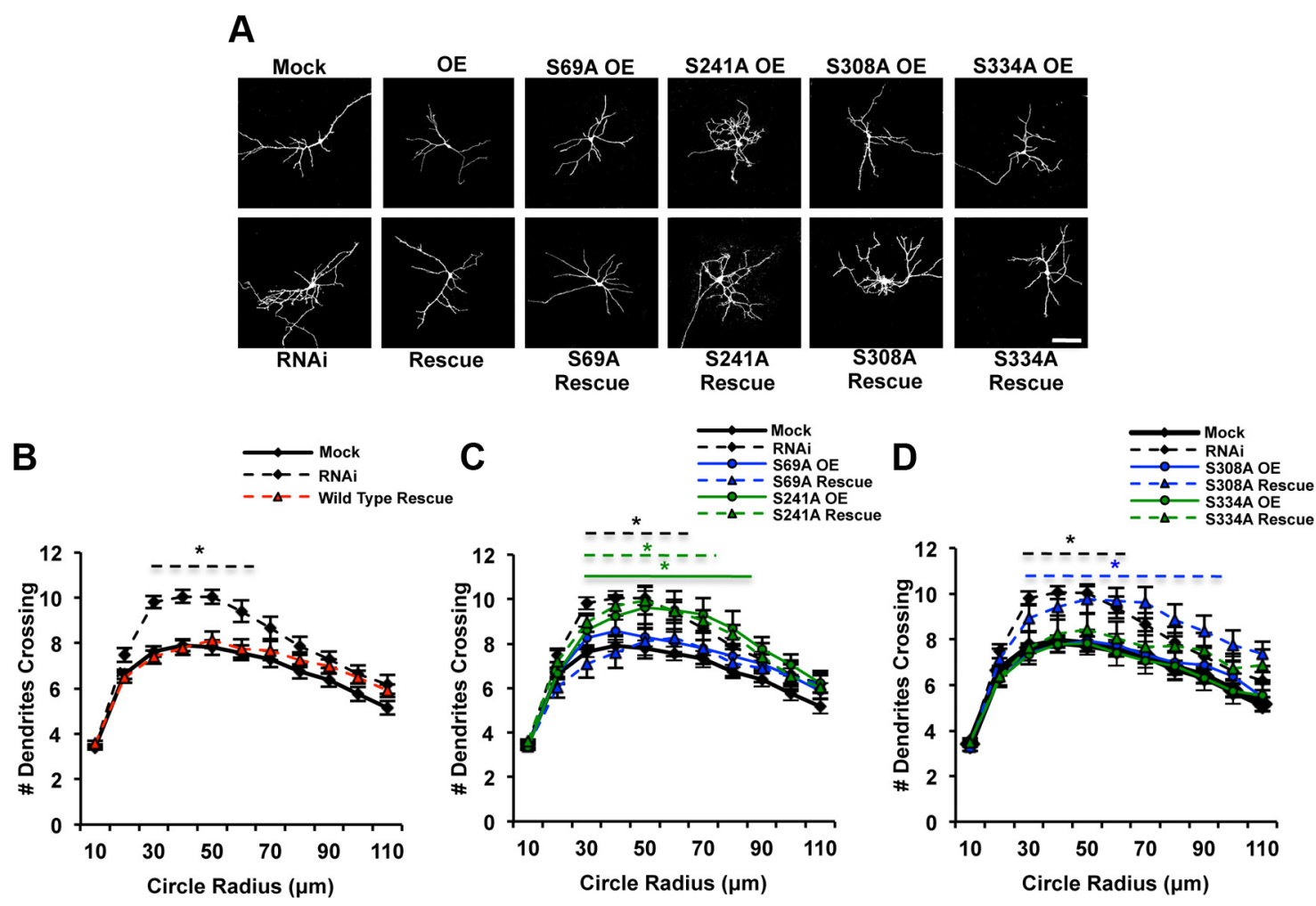

Figure 6. Serine 241 and 308 are required for Rem2 to mediate dendritic complexity. $A$, Representative images of 5 DIV mock and Rem2 RNAi-transfected neurons compared with neurons transfected with either an RNAi-resistant Rem2 CDNA alone [overexpression (OE)] or cotransfected with a Rem2 shRNA and an RNAi-resistant Rem2 (DNA (Rescue), as indicated. Note that overexpression of the "wild-type" RNAi-resistant Rem2 CDNA is shown here for comparison purposes only, as it has been extensively documented that overexpression of Rem2 is similar to wild type under these conditions (Ghiretti and Paradis, 2011), and thus this condition is not requantified in $\boldsymbol{B}-\boldsymbol{D}$. B, Quantification of dendritic complexity of 5 DIV mock transfected neurons compared with neurons transfected with either a Rem2 shRNA alone (RNAi) or a Rem2 shRNA and an RNAi-resistant Rem2 cDNA (Wild Type Rescue). C, Quantification of dendritic complexity of 5 DIV mock transfected neurons compared with neurons transfected with a Rem2 shRNA alone (RNAi), an RNAi-resistant Rem2 CDNA with serine to alanine mutation at S69 or S241 (0E), or a Rem2 shRNA and an RNAi-resistant Rem2 CDNA with serine-to-alanine mutation at S69 or S241 (Rescue). Mock and RNAi data from B are replotted for ease of comparison with other conditions. Note that S69A 0E and S69A Rescue are not significant from mock or wild-type rescue. D, Quantification of dendritic complexity of 5 DIV mock transfected neurons compared with neurons transfected with a Rem2 shRNA alone (RNAi), an RNAi-resistant Rem2 2 DNA with serine-to-alanine mutation at $\$ 308$ or S334 (OE), or a Rem2 shRNA and an RNAi-resistant Rem2 cDNA with serine-to-alanine mutation at S308 or S334 (Rescue). Mock and RNAi data from B) are replotted for ease of comparison with other conditions. Note that S308A OE, S334A OE, and S334A Rescue are not significant from mock or wild-type rescue. ${ }^{*} p<0.05$, multivariate ANOVA with Scheffé's post hoc test. $n=41-58$ neurons per condition.

In addition, overexpression of three mutants (S69, S308, and S334) produced phenotypes that were not significantly different from mock transfected neurons (Fig. $6 A, C, D$ ), as was previously observed with overexpression of a wild-type RNAi-resistant Rem2 cDNA (Ghiretti and Paradis, 2011). In contrast, the S241A RNAi-resistant Rem2 cDNA exhibited a dominantnegative effect when overexpressed, leading to an increase in complexity that was similar to that seen with RNAi-mediated knockdown of Rem2 (Fig. 6A,C). The dominant-negative phenotype observed with S241A suggests this site is a critical regulator of Rem 2 activity. Furthermore, the fact that the Rem2 RNAi plus Rem2 S241A dendritic complexity phenotype was equivalent to the phenotype of either Rem2 RNAi or Rem2 S241A alone suggests that the S241A mutation renders Rem2 a nonfunctional protein. Overall, these data are consistent with a model in which phosphorylation of Rem 2 by CaMKII at $\mathrm{S} 241$ and other sites activates Rem 2 signaling, which ultimately leads to the restriction of dendritic complexity.

\section{Phosphorylation by CaMKII affects nuclear localization} of Rem2

We next wondered what effect the phosphorylation by CaMKII at S241 and S308 might have on Rem2 function. We previously observed that Rem2 is widely expressed throughout hippocampal neurons, including the soma, dendrites, and nucleus (Ghiretti and Paradis, 2011). In our current study, we show that Rem2 directly or indirectly inhibits CaMKIV (Fig. 2), which is confined to the nucleus (Matthews et al., 1994), suggesting that nuclear localization of Rem 2 may be important for its function in this pathway. Therefore, we asked whether CaMKII-dependent phosphorylation of Rem 2 might alter its subcellular localization. To explore this hypothesis, we cotransfected neurons at 2 DIV with GFP and CA CaMKII $\alpha$ (T286D/T305A/T306A) (Pi et al., 2010), a Rem 2 cDNA containing both the S241A and S308A mutations, or both CA CaMKII $\alpha$ and the S241A/S308A Rem 2 cDNA. At 5 DIV, we fixed and labeled these neurons with DAPI (to label nuclei) and immunostained with either anti-Rem2 (to assess endogenous Rem 2 localization in response to CA CaMKII $\alpha$ expression) or anti-myc (to assess S241A/S2308A Rem2 localization). Then, we compared the nuclear versus cytoplasmic Rem2 localization for both endogenous Rem2 (which can be phosphorylated at S241 and S308) and S241A/S308A Rem2, in the presence or absence of constitutive CaMKII activity.

Interestingly, we observed that expression of CA CaMKII $\alpha$ caused enhanced nuclear localization of endogenous Rem2 (as assessed by the intensity of anti-Rem 2 immunostaining overlapping with DAPI) compared with a control condition in which CA CaMKII $\alpha$ was not expressed (Fig. 7A,C), suggesting that phosphorylation of Rem 2 by CaMKII regulates Rem 2 subcellular lo- 
A
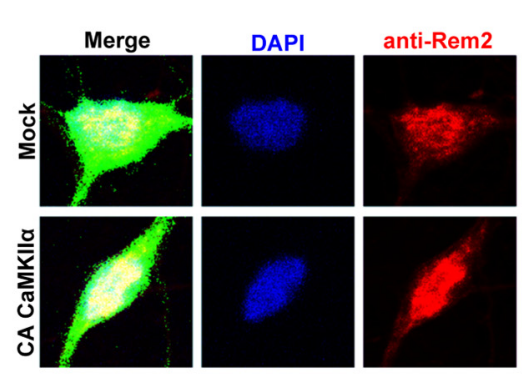

B
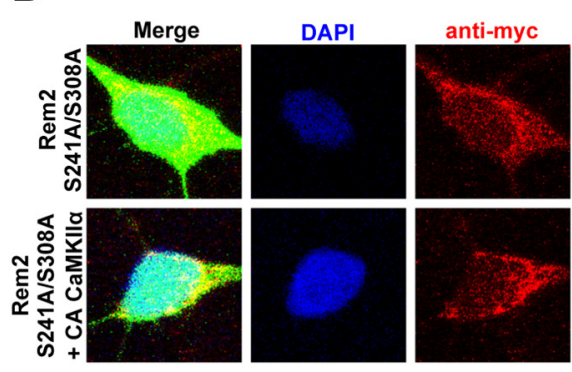
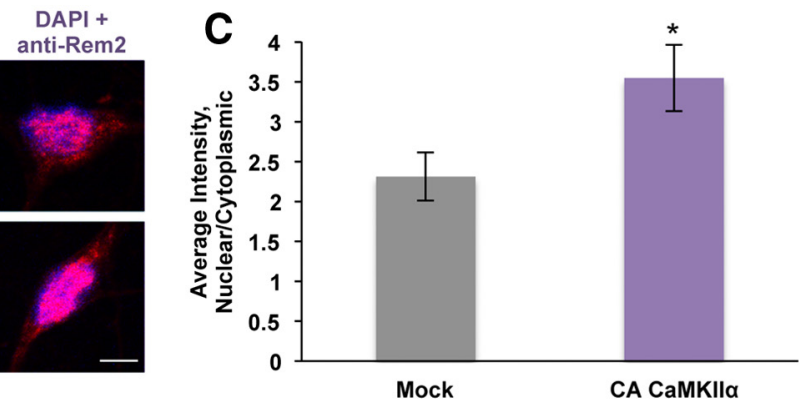

D
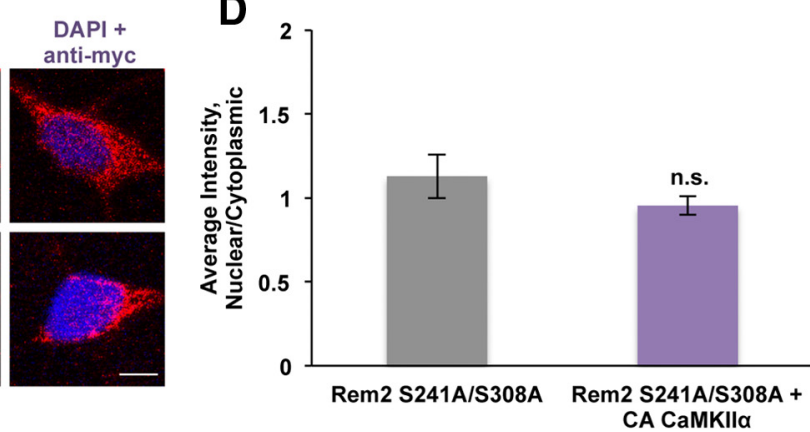

Figure 7. CaMKII phosphorylation leads to enhanced nuclear localization of Rem2.A, Representative images of the somas of 5 DIV mock transfected neurons compared with neurons transfected with CA CaMKIII. Neurons were labeled with DAPI (to label nuclei) and immunostained with anti-Rem2 (to label endogenous Rem2). Scale bar, $10 \mu \mathrm{m}$. B, Representative images of the somas of 5 DIV neurons transfected with Rem2 S241A/S308A compared with those transfected with Rem2 S241A/S308A and CA CaMKII. Neurons were labeled with DAPI and immunostained with anti-myc (to label Rem2 S241A/S308A). Scale bar, $10 \mu \mathrm{m}$. C, Quantification of the ratio of nuclear-to-cytoplasmic endogenous Rem2 expression. ${ }^{*} p<0.05$, pairwise ANOVA. $n>30$ neurons per condition. $D$, Quantification of the ratio of nuclear-to-cytoplasmic myc-tagged Rem2 S241A/S308A expression. There was no significance by pairwise ANOVA $(p=0.099) . n>30$ neurons per condition.

calization. In agreement with this hypothesis, CA CaMKII $\alpha$ expression had no effect on the localization of S241A/S308A Rem2 (Fig. $7 B, D$ ). This suggests that under normal conditions, the phosphorylation of Rem2 by CaMKII at S241 and/or S308 leads to the trafficking of Rem 2 to the nucleus. As the phosphorylation of these sites is critical for Rem 2 to limit dendritic complexity (Fig. 6), this also suggests that subcellular localization is an important mechanism through which the CaMKII/Rem2 signaling cascade is regulated.

\section{Discussion}

In this study, we demonstrate that Rem2 transduces CaMKdependent signals to instruct proper dendritic complexity and, furthermore, that Rem 2 and CaMK signaling can have opposing effects on dendritic arborization (Fig. 8). We also identify Rem2 as a novel CaMKII substrate in a signaling pathway that negatively regulates dendritic complexity. Our analysis specifically identifies S241 and S308 as key residues that regulate Rem2 activity via CaMKII phosphorylation. Furthermore, the phosphorylation of these serines by CaMKII leads to enhanced nuclear localization of Rem2, providing a novel mechanism through which Rem 2 activity is regulated.

Our epistatic and biochemical analyses are most consistent with a model that places Rem 2 downstream of CaMKII and upstream of CaMKIV (Fig. 8A). Although it is unclear whether Rem2 lies directly upstream of CaMKIV, both proteins are present in the nucleus (Matthews et al., 1994; Béguin et al., 2005; Mahalakshmi et al., 2007; Ghiretti and Paradis, 2011; Fig. 7). It is known that a major function of nuclear CaMKIV is to phosphorylate and activate the transcription factor CREB, promoting $\mathrm{Ca}^{2+}$-dependent transcription (Matthews et al., 1994; Sun et al., 1994) of genes that promote dendritic complexity (Redmond et al., 2002). Whereas the function of nuclear Rem 2 remains obscure, our study suggests the possibility that Rem 2 and CaMKIV may interact in the nucleus. Recently, the localization of overexpressed and fluorescently tagged Rem 2 was shown to shift from a diffuse to punctate pattern in cultured hippocampal neurons after neuronal depolarization, and pharmacological inhibition of CaMKII blocks this redistribution (Flynn et al., 2012). Furthermore, this study demonstrated that Rem 2 and CaMKII can coimmunoprecipitate in a complex with CaM from heterologous cells (Flynn et al., 2012). Our findings demonstrate that Rem2 represents a novel effector through which CaMKII regulates dendritic morphology.

Together, these data suggest the following model with respect to the relationship between CaMKII, Rem2, and CaMKIV. We hypothesize that after neuronal depolarization, Rem2 and activated CaMKII coassociate, allowing CaMKII to phosphorylate Rem 2 at S241 and S308 (Fig. 8 A). This phosphorylation leads to nuclear trafficking of Rem2, where it either directly or indirectly inhibits the activity of CaMKIV (Fig. $8 \mathrm{~A}$ ), which normally activates CREB-dependent transcription of genes that promote dendritic complexity. Thus, phospho-Rem2 in the nucleus is required to inhibit dendritic complexity. Furthermore, our model provides an explanation for a puzzling observation: thus far, we have not found conditions in which overexpression of wild-type Rem 2 limits dendritic complexity (Ghiretti and Paradis, 2011). We postulate that the lack of an observable Rem2 overexpression phenotype is attributable to a requirement for activated CaMKII in the phosphorylation and subsequent activation of Rem2. Thus, overexpression of Rem 2 in the absence of increased CaMKII activity has no effect. Our finding that Rem2 S241A overexpression exerts a dominant-negative effect on dendritic complexity (Fig. 6) also supports this hypothesis.

Previously, Rem 2 phosphorylation was primarily studied in non-neuronal cell types in the context of Rem2/14-3-3 protein dimer interactions, for which simultaneous phosphorylation of 
S69 and S334 is required (Béguin et al., 2005). Simultaneous mutation of S69 and S334 to alanines disrupts the ability of Rem 2 to inhibit dendritic complexity and leads to enhanced nuclear localization of Rem2, although this nuclear Rem2 is not able to inhibit dendritic complexity (Ghiretti and Paradis, 2011), presumably because it has not been phosphorylated by CaMKII on S241 and/or S308. Our current study shows that mutation of either S69 or S334 on its own does not affect the ability of Rem 2 to mediate dendritic complexity (Fig. 6). One possible explanation for this finding is that 14-3-3 binding can still occur in the absence of one of these serine residues, as has been shown previously (Béguin et al., 2005), leading us to speculate that even a reduced Rem2/143-3 interaction is still sufficient to regulate dendritic complexity.

Rem 2 contains a putative nuclear localization sequence (NLS) at its C terminus (Fig. 8 B, gray box; amino acids $306-$ 328 , as identified by http://nls-mapper. iab.keio.ac.jp/); interestingly, S308 is found immediately at its start (Fig. $8 \mathrm{~B}$ ). It is known that phosphorylation of sites located near the start of an NLS can lead to conformational changes that expose the NLS to importins, thus enhancing nuclear import (Nardozzi et al., 2010). Our data are consistent with the hypothesis that phosphorylation of S308 and/or S241 exposes the NLS of Rem 2 to the relevant importin, which allows Rem 2 to be trafficked to the nucleus (Fig. $8 \mathrm{~A}$ ). In the absence of phosphorylation at these sites, Rem 2 remains in the cytoplasm, perhaps due to the phosphorylation of S69 and S334 and subsequent 14-3-3 protein binding (Ghiretti and Paradis, 2011), as the NLS is not accessible. It will be interesting to determine whether the putative NLS is necessary and sufficient for Rem 2 localization.

Overall, our studies suggest that Rem2 is regulated through posttranslational modification (e.g., phosphorylation by CaMKII), rather than by its nucleotide binding state as is true for most GTPases. This characteristic may be a defining feature of the RGK family, as comparison of the amino acid sequence of mouse RGK family members reveals excellent conservation of the S241, S308, and S334 phosphorylation sites, as well as the putative NLS (Fig. $8 B$ ). Previously, a number of overexpression studies have implicated RGK family members as negative regulators of calcium channel function (Finlin et al., 2003, 2005; Ward et al., 2004; Chen et al., 2005). Although our study does not address a role for Rem2 in calcium channel function, it is interesting to speculate that Rem 2 could act in a negative feedback loop to turn off $\mathrm{Ca}^{2+}$ mediated signaling that instructs the dendritic arbor. However, additional studies are needed to determine whether an endogenous function of Rem 2 and other RGK family members is to regulate calcium channel function. Nonetheless, it is clear from our analysis of Rem $2 / \mathrm{CaMK}$ interactions that there are diverse and potentially distinct activity-dependent signaling pathways that synergize to regulate dendritic complexity.

\section{References}

Ageta-Ishihara N, Takemoto-Kimura S, Nonaka M, Adachi-Morishima A, Suzuki K, Kamijo S, Fujii H, Mano T, Blaeser F, Chatila TA, Mizuno H, Hirano T, Tagawa Y, Okuno H, Bito H (2009) Control of cortical axon elongation by a GABA-driven Ca2+/calmodulin-dependent protein kinase cascade. J Neurosci 29:13720-13729. CrossRef Medline

Béguin P, Mahalakshmi RN, Nagashima K, Cher DH, Kuwamura N, Yamada Y, Seino Y, Hunziker W (2005) Roles of 14-3-3 and calmodulin binding in subcellular localization and function of the small G-protein Rem2. Biochem J 390:67-75. CrossRef Medline

Béguin P, Ng YJ, Krause C, Mahalakshmi RN, Ng MY, Hunziker W (2007) RGK small GTP-binding proteins interact with the nucleotide kinase domain of $\mathrm{Ca} 2+$-channel beta-subunits via an uncommon effector binding domain. J Biol Chem 282:11509-11520. CrossRef Medline

Chang BH, Mukherji S, Soderling TR (1998) Characterization of a calmodulin kinase II inhibitor protein in brain. Proc Natl Acad Sci U S A 95: 10890-10895. CrossRef Medline

Chen H, Puhl HL 3rd, Niu SL, Mitchell DC, Ikeda SR (2005) Expression of Rem2, an RGK family small GTPase, reduces N-type calcium current without affecting channel surface density. J Neurosci 25:9762-9772. CrossRef Medline

Correll RN, Pang C, Niedowicz DM, Finlin BS, Andres DA (2008) The RGK family of GTP-binding proteins: regulators of voltage-dependent calcium channels and cytoskeleton remodeling. Cell Signal 20:292-300. CrossRef Medline

Enslen H, Sun P, Brickey D, Soderling SH, Klamo E, Soderling TR (1994) 
Characterization of Ca2 +/calmodulin-dependent protein kinase IV. Role in transcriptional regulation. J Biol Chem 269:15520-15527. Medline

Fink CC, Bayer KU, Myers JW, Ferrell JE Jr, Schulman H, Meyer T (2003) Selective regulation of neurite extension and synapse formation by the beta but not the alpha isoform of CaMKII. Neuron 39:283-297. CrossRef Medline

Finlin BS, Shao H, Kadono-Okuda K, Guo N, Andres DA (2000) Rem2, a new member of the Rem/Rad/Gem/Kir family of Ras-related GTPases. Biochem J 347:223-231. CrossRef Medline

Finlin BS, Crump SM, Satin J, Andres DA (2003) Regulation of voltagegated calcium channel activity by the Rem and Rad GTPases. Proc Natl Acad Sci U S A 100:14469-14474. CrossRef Medline

Finlin BS, Mosley AL, Crump SM, Correll RN, Ozcan S, Satin J, Andres DA (2005) Regulation of L-type Ca2 + channel activity and insulin secretion by the Rem2 GTPase. J Biol Chem 280:41864-41871. CrossRef Medline

Flynn R, Labrie-Dion E, Bernier N, Colicos MA, De Koninck P, Zamponi GW (2012) Activity-dependent subcellular cotrafficking of the small GTPase Rem2 and $\mathrm{Ca} 2+/ \mathrm{CaM}$-dependent protein kinase IIalpha. PLoS One 7:e41185. CrossRef Medline

Gao L, Blair LA, Marshall J (2006) CaMKII-independent effects of KN93 and its inactive analog KN92: reversible inhibition of L-type calcium channels. Biochem Biophys Res Commun 345:1606-1610. CrossRef Medline

Gaudillière B, Konishi Y, de la Iglesia N, Yao GI, Bonni A (2004) A CaMKIINeuroD signaling pathway specifies dendritic morphogenesis. Neuron 41:229-241. CrossRef Medline

Ghiretti AE, Paradis S (2011) The GTPase Rem2 regulates synapse development and dendritic morphology. Dev Neurobiol 71:374-389. CrossRef Medline

Horton AC, Ehlers MD (2003) Neuronal polarity and trafficking. Neuron 40:277-295. CrossRef Medline

Jan YN, Jan LY (2003) The control of dendrite development. Neuron 40: 229-242. CrossRef Medline

Kennelly PJ, Krebs EG (1991) Consensus sequences as substrate specificity determinants for protein kinases and protein phosphatases. J Biol Chem 266:15555-15558. Medline

Ledoux J, Chartier D, Leblanc N (1999) Inhibitors of calmodulindependent protein kinase are nonspecific blockers of voltage-dependent K+ channels in vascular myocytes. J Pharmacol Exp Ther 290:1165-1174. Medline

Lisman J, Schulman H, Cline H (2002) The molecular basis of CaMKII function in synaptic and behavioural memory. Nat Rev Neurosci 3:175190. CrossRef Medline

Lisman J, Yasuda R, Raghavachari S (2012) Mechanisms of CaMKII action in long-term potentiation. Nat Rev Neurosci 13:169-182. CrossRef Medline

Loebrich S, Nedivi E (2009) The function of activity-regulated genes in the nervous system. Physiol Rev 89:1079-1103. CrossRef Medline

Lyons MR, West AE (2011) Mechanisms of specificity in neuronal activityregulated gene transcription. Prog Neurobiol 94:259-295. CrossRef Medline

Mahalakshmi RN, Ng MY, Guo K, Qi Z, Hunziker W, Béguin P (2007) Nuclear localization of endogenous RGK proteins and modulation of cell shape remodeling by regulated nuclear transport. Traffic 8:1164-1178. CrossRef Medline

Matthews RP, Guthrie CR, Wailes LM, Zhao X, Means AR, McKnight GS (1994) Calcium/calmodulin-dependent protein kinase types II and IV differentially regulate CREB-dependent gene expression. Mol Cell Biol 14:6107-6116. CrossRef Medline

Moyers JS, Bilan PJ, Zhu J, Kahn CR (1997) Rad and Rad-related GTPases interact with calmodulin and calmodulin-dependent protein kinase II. J Biol Chem 272:11832-11839. CrossRef Medline

Moyers JS, Zhu J, Kahn CR (1998) Effects of phosphorylation on function of the Rad GTPase. Biochem J 333:609-614. Medline

Nardozzi JD, Lott K, Cingolani G (2010) Phosphorylation meets nuclear import: a review. Cell Commun Signal 8:32. CrossRef Medline
Paradis S, Harrar DB, Lin Y, Koon AC, Hauser JL, Griffith EC, Zhu L, Brass LF, Chen C, Greenberg ME (2007) An RNAi-based approach identifies molecules required for glutamatergic and GABAergic synapse development. Neuron 53:217-232. CrossRef Medline

Pearson RB, Kemp BE (1991) Protein kinase phosphorylation site sequences and consensus specificity motifs: tabulations. Methods Enzymol 200:62-81. CrossRef Medline

Pi HJ, Otmakhov N, Lemelin D, De Koninck P, Lisman J (2010) Autonomous CaMKII can promote either long-term potentiation or long-term depression, depending on the state of T305/T306 phosphorylation. J Neurosci 30:8704-8709. CrossRef Medline

Puram SV, Kim AH, Ikeuchi Y, Wilson-Grady JT, Merdes A, Gygi SP, Bonni A (2011) A CaMKIIbeta signaling pathway at the centrosome regulates dendrite patterning in the brain. Nat Neurosci 14:973-983. CrossRef Medline

Redmond L, Kashani AH, Ghosh A (2002) Calcium regulation of dendritic growth via CaM kinase IV and CREB-mediated transcription. Neuron 34:999-1010. CrossRef Medline

Reymond P, Coquard A, Chenon M, Zeghouf M, El Marjou A, Thompson A, MénétreyJ (2012) Structure of the GDP-bound G domain of the RGK protein Rem2. Acta Crystallogr Sect F Struct Biol Cryst Commun 68:626631. CrossRef Medline

Sasson Y, Navon-Perry L, Huppert D, Hirsch JA (2011) RGK family Gdomain:GTP analog complex structures and nucleotide-binding properties. J Mol Biol 413:372-389.

Schmitt JM, Wayman GA, Nozaki N, Soderling TR (2004) Calcium activation of ERK mediated by calmodulin kinase I. J Biol Chem 279:2406424072. CrossRef Medline

Sholl DA (1953) Dendritic organization in the neurons of the visual and motor cortices of the cat. J Anat 87:387-406. Medline

Sun P, Enslen H, Myung PS, Maurer RA (1994) Differential activation of CREB by $\mathrm{Ca} 2+/$ calmodulin-dependent protein kinases type II and type IV involves phosphorylation of a site that negatively regulates activity. Genes Dev 8:2527-2539. CrossRef Medline

Takemoto-Kimura S, Terai H, Takamoto M, Ohmae S, Kikumura S, Segi E, Arakawa Y, Furuyashiki T, Narumiya S, Bito H (2003) Molecular cloning and characterization of CLICK-III/CaMKIgamma, a novel membrane-anchored neuronal $\mathrm{Ca} 2+/$ calmodulin-dependent protein $\mathrm{ki}$ nase (CaMK). J Biol Chem 278:18597-18605. CrossRef Medline

Vaillant AR, Zanassi P, Walsh GS, Aumont A, Alonso A, Miller FD (2002) Signaling mechanisms underlying reversible, activity-dependent dendrite formation. Neuron 34:985-998. CrossRef Medline

Ward Y, Spinelli B, Quon MJ, Chen H, Ikeda SR, Kelly K (2004) Phosphorylation of critical serine residues in Gem separates cytoskeletal reorganization from down-regulation of calcium channel activity. Mol Cell Biol 24:651-661. CrossRef Medline

Wayman GA, Kaech S, Grant WF, Davare M, Impey S, Tokumitsu H, Nozaki N, Banker G, Soderling TR (2004) Regulation of axonal extension and growth cone motility by calmodulin-dependent protein kinase I. J Neurosci 24:3786-3794. CrossRef Medline

Wayman GA, Impey S, Marks D, Saneyoshi T, Grant WF, Derkach V, Soderling TR (2006) Activity-dependent dendritic arborization mediated by CaM-kinase I activation and enhanced CREB-dependent transcription of Wnt-2. Neuron 50:897-909. CrossRef Medline

Wayman GA, Tokumitsu H, Davare MA, Soderling TR (2011) Analysis of CaM-kinase signaling in cells. Cell Calcium 50:1-8. CrossRef Medline

Wheeler DG, Barrett CF, Groth RD, Safa P, Tsien RW (2008) CaMKII locally encodes L-type channel activity to signal to nuclear CREB in excitation-transcription coupling. J Cell Biol 183:849-863. CrossRef Medline

Wu GY, Cline HT (1998) Stabilization of dendritic arbor structure in vivo by CaMKII. Science 279:222-226. CrossRef Medline

Xia Z, Dudek H, Miranti CK, Greenberg ME (1996) Calcium influx via the NMDA receptor induces immediate early gene transcription by a MAP kinase/ERK-dependent mechanism. J Neurosci 16:5425-5436. Medline 